\title{
Confiança organizacional como chave para a coordenação implícita e para a construção do capital intangível
}

\section{Organizational Trust as the Key to Implicit Coordination and to build Intangible Capital}

Marco Zanini ${ }^{1}$

"Há um elemento de confiança em toda a transação"

(ARROW, 1973, p.24)

\section{Resumo}

Este artigo busca contribuir para as discussões sobre o tema confiança interpessoal dentro das organizações numa perspectiva da Economia Organizacional, introduzindo confiança como elemento central para a coordenação implícita das tarefas organizacionais e a sua relação com o desempenho organizacional. Entendo confiança interpessoal como um elemento central para o melhor entendimento de contratos relacionais, este artigo ilustra a sua relevância para a coordenação e para o desempenho das diversas tarefas organizacionais.

Palavras-chave: Confiança, Coordenação Implicita, Capital Intangivel.

\begin{abstract}
This article provides a contribution to the discussions about the theme interpersonal trust within organizations in the Organizational Economics perspective. It introduces trust as a central element to the implicit coordination of organizational tasks and its relation with organizational performance. I understand interpersonal trust as a central element for a better understanding of relational contracts. This article illustrates the relevance of trust to the coordination and performance of organizational tasks.
\end{abstract}

Keywords: Trust, Implicit Coordination, Intangible Capital

\section{Introdução}

A existência de confiança entre os membros de uma empresa pode contribuir de forma implícita para aumentar significativamente a eficiência na coordenação das diversas tarefas organizacionais. O aumento da eficiência das transações econômicas internas à organização, por meio de um mecanismo contratual implícito, implica a construção de um capital intangível. Em muitos casos, a presença de confiança entre os membros corporativos poderá assumir a forma de um diferencial competitivo ou de uma competência organizacional distinta. A lógica dessa função está na forma como esse mecanismo informal opera em contratos relacionais. Onde existir a chance de indivíduos interagirem repetidamente, ao longo do tempo, assimetrias de tempo de entrega trazem risco à transação para a parte que precisa investir antes de receber algo em retorno, e dessa forma, certo grau de

\footnotetext{
'Marco.Zanini@Student.Uni-Magdeburg.DE - Professor da Fundação Dom Cabral (FDC) e Doutor na Universidade Otto-von-Guericke Magdeburg, Alemanha Rua Francisco Otaviano 60 / 206 - Copacabana - Rio de Janeiro - RJ - Brasil - CEP: 22080-040.

Artigo recebido em agosto de 2006 e aceito para publicação em outubro de 2006
} 
confiança é necessário. Essa relação bilateral torna a confiança o elemento central a ser analisado em contratos relacionais.

Contratos de trabalho são exemplos de contratos relacionais baseados em confiança interpessoal. Mesmo que esses contratos necessitem ser firmados por meio de contratos explícitos - na forma de um documento formal por razões legais do arcabouço institucional, estes se tornam contratos relacionais entre agentes de interação ao longo do tempo. Ao invés de antecipar todas as contingências futuras, contratos relacionais antecipam uma série de incertezas, na forma de transações entre os parceiros de interatividade, durante um longo período. Por isso, esses contratos são incompletos por definição. Os problemas contratuais que porventura possam surgir numa relação de trabalho podem ser reduzidos de forma significante com a presença de confiança. A confiança que poderá ser construída como fruto da interação entre os agentes funciona como um mecanismo implícito de controle e coordenação para diversas atividades organizacionais. Para exemplificar, tomamos $\mathbf{A}$ e $\mathbf{B}$ como agentes interativos. Vamos assumir que A deverá realizar um investimento de confiança em B. Quando B aceita voluntariamente esse investimento de confiança realizado por $\mathbf{A}, \mathbf{B}$ estabelece um contrato implícito entre ambos, de forma a atender as expectativas de $\mathbf{A}$. O atendimento das expectativas de $\mathbf{A}$ por $\mathbf{B}$ é objeto desse contrato implícito. A realização dessa transação baseada na cooperação voluntária entre esses agentes cria uma relação de interdependência que por sua vez reduzirá o risco relativo para a realização de uma determinada tarefa organizacional. Assim, a confiança interpessoal presente nas estruturas hierárquicas funciona como um "lubrificante" para as diversas transações econômicas que ocorrem entre os agentes corporativos, por exemplo, facilitando a comunicação organizacional e a transferência do conhecimento, o que por fim poderá representar um desempenho organizacional superior.

Neste artigo analisamos o elemento confiança, sua relação com a coordenação implícita e, posteriormente, a sua relação com o desempenho organizacional. Para melhor compreender esse mecanismo social e as suas consequiências dentro das organizações, apresentamos este artigo em três seções: Na primeira seção definimos e ilustramos o elemento confiança como um mecanismo de coordenação implícita dentro das organizações. Em seguida, apresentamos uma definição e análise dos contratos relacionais tendo confiança como seu elemento central. Na ultima seção apresentamos a relação entre confiança organizacional e o desempenho organizacional.

\section{Confiança como um mecanismo de coordenação implícita}

Hardin (1998) afirma que relacionamentos de confiança são compostos de três partes: 1) as características pessoais de quem realiza um investimento de confiança - aqui denominamos o indivíduo que confia de $\mathbf{A}$;2) as características pessoais de quem recebe o investimento de confiança - aqui denominamos o indivíduo que recebe o investimento de confiança de $\mathbf{B} ; 3$ ) o específico contexto transacional onde ocorre uma determinada relação de confiança - denominamos esse contexto específico de $\mathbf{X}$.

De acordo com Hardin (2002), a confiança consiste em um "interesse encapsulado", no sentido de que as expectativas do indivíduo que confia (A) estão baseadas na percepção que este possui das motivações do indivíduo que recebe o investimento de confiança (B). Assim, mesmo que esses indivíduos possuam certas divergências em incentivos e interesses, a existência de algum ponto comum de interesse poderá estimular $\mathbf{A}$ a realizar um investimento de confiança em $\mathbf{B}$. Com isso, dependendo das pessoas que interagem e do contexto específico, uma relação de confiança pode se estabelecer em diferentes bases, de formas diferentes e em níveis diferentes. Podemos confiar em uma pessoa de forma constante, para várias questões, ou podemos confiar em outra pessoa uma única vez, somente para algumas questões.

Na mesma linha de Hardin, Ripperger (1998) apresenta um trabalho que vai além da pura teoria da decisão racional para construir um melhor entendimento sobre as relações de confiança, incluindo alguns pressupostos na forma de estados psicológicos. Ripperger (1998, p.45.) contribui para um melhor entendimento sobre os investimentos de confiança em ações cooperativas assumindo que duas condições precisam ser satisfeitas para haver ações cooperativas baseadas em confiança: a coexistência de uma expectativa de confiança e uma ação baseada em confiança. A existência desses dois componentes centrais irá alicerçar ações baseadas em confiança. Segundo Ripperger, uma expectativa de confiança é definida como a expectativa de quem confia (A) em outra pessoa $(\mathbf{B})$, acreditando que $(\mathbf{B})$ estará motivada a não agir de uma forma oportunista. Assim, uma 
ação baseada em confiança é um investimento voluntário da pessoa que confia (A), na forma de ação concreta, sob situação de risco comportamental, sem, no entanto, recorrer a nenhum mecanismo explícito de segurança.

Ainda conforme essa análise, a confiança pode ser entendida como uma predisposição ou expectativa que leve a uma ação calculada envolvendo risco comportamental. Tal expectativa se manifesta através de um estado subjetivo inicial, ou de uma reação emocional, traduzido em uma condição cognitiva racional. Dessa forma, um indivíduo consegue avaliar e calcular sua predisposição a se engajar numa determinada situação que envolva um risco relativo ao comportamento de outro. Ripperger (1998, p.45) define confiança como aceitação voluntária e antecipada de um investimento de risco, pela abdicação de mecanismos contratuais explícitos de segurança e de controle contra comportamentos oportunistas, na expectativa de que a outra parte não agirá dessa forma.

Como Hardin, Ripperger (1998) entende que uma expectativa de confiança, que é o pressuposto para uma ação concreta de confiança, baseia-se na percepção subjetiva do indivíduo que confia (A) sobre as motivações (autointeresses percebidos) do indivíduo que recebe o investimento de confiança (B), em corresponder ao seu investimento. Assim Hardin (2002, p.3.) entende que o indivíduo que recebe o investimento de confiança (B) "encapsula" os interesses do indivíduo que confia (A), quando este honra o investimento confiado.

De acordo com Ripperger (1998, p.92-98.), a expectativa de confiança, na forma de um estado psicológico, possui dois elementos de forma dualística: um aspecto emocional e um aspecto cognitivo. Esses dois componentes são coexistentes e podem prevalecer um sobre o outro, de acordo com as pessoas com quem interagimos e as situações específicas. Por vezes, uma expectativa de confiança estará baseada mais em aspectos emocionais; outras vezes, essa expectativa estará baseada mais em aspectos cognitivos. Dessa forma, agentes de interação poderão estar motivados de forma diferente, sobre bases mais emocionais ou mais cognitivas, de acordo com uma determinada pessoa ou grupo de pessoas, situações, contextos ou circunstâncias.

Ripperger (1998, p.97.) comenta que a base cognitiva traz às expectativas do indivíduo que confia (A) a capacidade de calcular o risco relativo em uma determinada situação. A autora define a parte cognitiva como confiança cognitiva ou confiança calculada (kalkulierendes Vertrauen). Por outro lado, a base emocional traz aspectos afetivos e emocionais às expectativas do indivíduo que confia (A) que não podem ser removidos da análise de um investimento de confiança porque trazem em si um aspecto fundamental e inerente a uma relação de confiança: uma crença. Essa crença é necessária para superar a informação cognitiva imperfeita causada pelas incertezas de uma penetração intelectual limitada. No entanto, Ripperger observa que os elementos emocionais são reações desenvolvidas sobre uma compreensão cognitiva. Sentimentos pessoais construídos sob reações emocionais são desenvolvidos sobre uma compreensão cognitiva relativa às específicas situações. Pelo fato das emoções desenvolverem-se como reações sobre estruturas cognitivas em situações específicas, possuem para nós um caráter compreensivo. Assim, embora cada expectativa de confiança traga em si aspectos cognitivos e emocionais, os aspectos cognitivos são preponderantes na análise das motivações e reações do indivíduo que recebe o investimento de confiança (B). Conseqüentemente, uma vez que uma relação de confiança é atribuída basicamente às motivações de $\mathbf{B}$ (pela percepção subjetiva de $\mathbf{A}$ ), cada evento de confiança deve, antes de tudo, reconhecer os aspectos cognitivos das motivações de $\mathbf{B}$, mesmo que sob fortes circunstâncias emocionais (Ripperger, p.98.). A construção da expectativa de confiança é baseada na percepção subjetiva de $\mathbf{A}$ das motivações de $\mathbf{B}$ em honrar ou desonrar o seu investimento de confiança (ver figura 1). 


\section{Figura 1 - Expectativa de confiança como percepção subjetiva das motivações de quem faz um investimento de confiança}

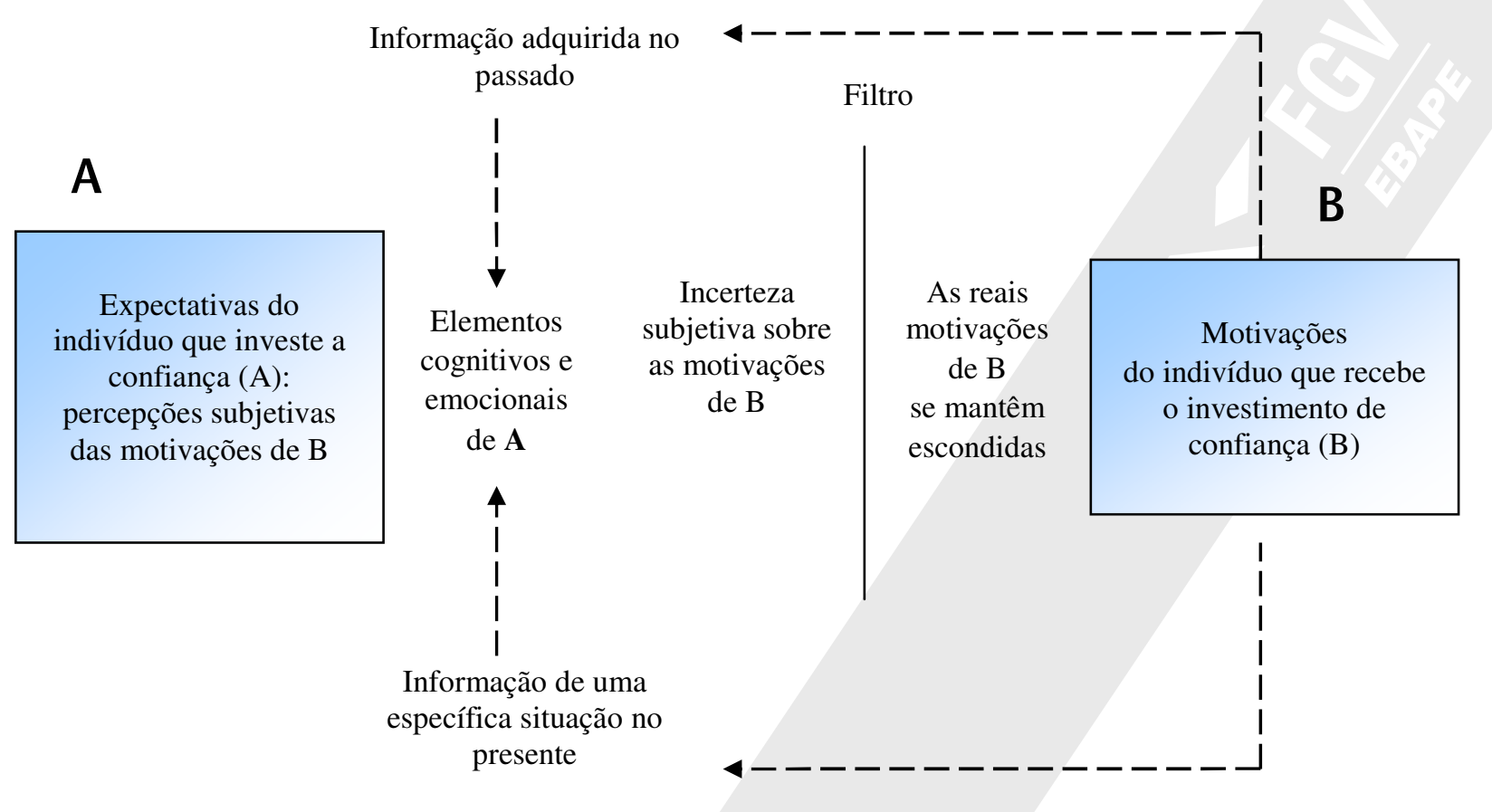

Fonte: Ripperger (1998, p.95).

As estruturas cognitivas e emocionais de A são influenciadas pelas formas de percepção e característica objetivas de uma determinada situação, pela informação adquirida de interações com $\mathbf{B}$ no passado (em forma de reputação) e pelas informações sobre a específica situação no presente. Ripperger (1998, p.65.) observa assim um "filtro" que divide a percepção de A das motivações de $\mathbf{B}$ e as reais motivações de $\mathbf{B}$. Com isso podemos reconhecer aqui o conceito de informação assimétrica nas relações de confiança. No entanto, o problema crucial nas relações de confiança não é a informação incompleta sobre a confiabilidade da outra pessoa, mas sim a nossa ignorância sobre os limites dessa confiabilidade. Podemos entender que realizamos uma avaliação de outras pessoas, ou grupo de pessoas, baseados na informação limitada que temos sobre suas motivações (como "interesses encapsulados") em agir de forma a honrar o nosso investimento de confiança.

A expectativa de confiança existe potencialmente mesmo antes de se realizar uma ação concreta baseada nessa expectativa (ver figura 2). Essa expectativa continua existindo durante o curso de uma ação baseada em confiança, durante o surgimento dos resultados esperados e mesmo após a situação crítica de risco comportamental, inerente a uma relação de confiança. Por essa razão podemos entender que A poderá confiar em B independente da realização de uma ação concreta baseada nessa confiança (em T1). Assim, dizemos que A possui certa predisposição de se engajar voluntariamente em uma específica situação de risco com $\mathbf{B}$, independente das chances de interação entre ambos. Dessa forma, confiança pode ser entendida como um elemento de motivação implícito para uma possível ação concreta de confiança. 


\section{Figura 2 - Investimento de confiança e a presença de risco comportamental}

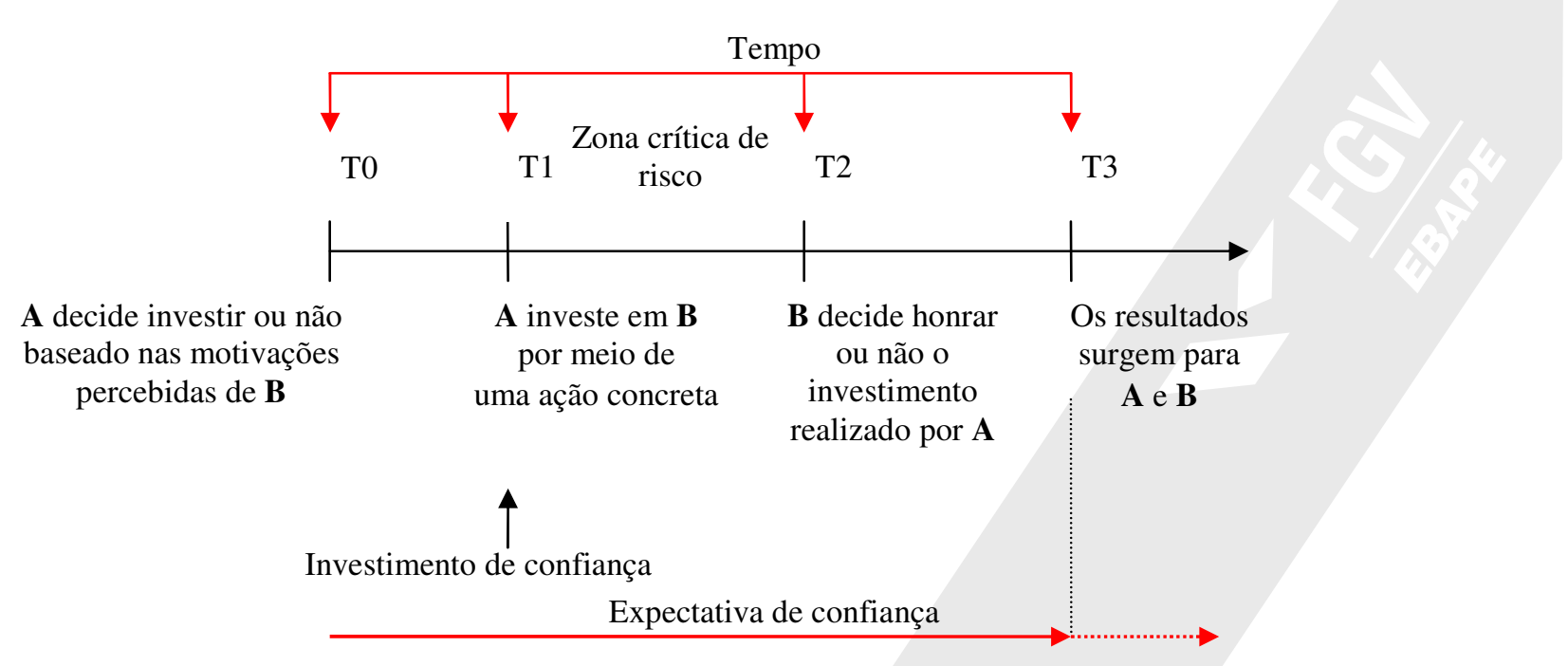

Fonte: o autor

\section{Contratos relacionais baseados em confiança}

Analisado o mecanismo confiança, passamos nesta seção a melhor compreender a sua função nos contratos relacionais. Confiança é o elemento central para uma melhor avaliação dos contratos relacionais (CASSON, 1997, p.7-9, GIBBONS, 2000, 2001; KREPS, 1990; WOLFF, 1996, p.102-105). ${ }^{1}$

Na perspectiva econômica, contratos relacionais, como contratos de trabalho, são contratos incompletos usados para explicar a cooperação num mundo de eventos futuros incertos (FURUBOTN; RICHTER, 2001, p.175 e 265.). Por definição, contratos relacionais referem-se a relações contratuais de longo prazo, de forma que, qualquer nova informação que se faça disponível, exogenamente ou endogenamente ao sistema, poderá gerar novas opções para os parceiros de interação na forma de novos comportamentos (WOLFF, 1995, p.91.). Dessa forma, pode ser vantajoso para parceiros de interação incorporar essa nova informação ao relacionamento, buscando assim um equilíbrio seqüencial no tempo. ${ }^{2}$

Por definição, contratos relacionais não podem ser monitorados ou controlados por terceiros. Rearranjos contratuais são necessários ao longo do tempo, de forma a assegurar um processo de cooperação contínuo entre os parceiros de interação (WOLFF, 1995, p.91.). De acordo com o contexto específico, confiança como um mecanismo de controle pode ser altamente desejável e eficiente para lidar com as incertezas comportamentais causadas pelo surgimento de nova informação nas relações entre parceiros de interação. Quanto maior a freqüência do surgimento de nova informação dentro do sistema, maiores serão as chances do aumento da incerteza comportamental, e mais necessário será o uso de contratos relacionais baseados em confiança como forma de lidar com essa incerteza.

Dessa forma, o modo como a relevância e a eficiência da confiança atuam como um mecanismo de governança está essencialmente relacionado com o grau de incerteza endógena e exógena, seja pela falta ou pela confiabilidade da informação ou pela frequiência com que o surgimento de nova informação dentro do sistema modifica o comportamento das pessoas. Além disso, mecanismos sociais como confiança são particularmente importantes quando o término de um relacionamento representa um custo muito alto (particularmente nos relacionamentos onde existe uma relação específica construída ao longo do tempo). 
Contratos de trabalho são exemplos de contratos relacionais baseados em relacionamentos específicos. Estes podem combinar as características formais de contratos legais, devido às exigências institucionais, e as características de contratos informais, na forma de reputações construídas dentro de relacionamentos idiossincráticos. Contratos de trabalho estão baseados em mecanismos que determinam o seu cumprimento (self-enforcing), de forma a assegurar a eficiência dessas relações, exatamente, pela dificuldade de monitoração e avaliação que apresentam (KEEFER; KNACK, 2005, p.706.). Dessa forma, eles podem ser mais bem compreendidos pelo modelo de reputações do comportamento racional confrontado por contingências imprevisíveis (ver KREPS, 1990; WOLFF, 1996). Nesse caso, a confiança gerada nos relacionamentos interpessoais funciona como um mecanismo de autocontrole, presente em determinado grau em todas as relações de trabalho. A lógica reside no fato de que as partes concordam antecipadamente em cooperar no longo prazo porque ambas querem manter sua reputação para as futuras transações. Há algum tempo, Macauley (1963, p.62.) já afirmava que os homens de negócio buscam manter seu comprometimento baseado em sua reputação e amizades pessoais.

Numa abordagem econômica, a premissa central dos contratos relacionais baseados em confiança é a de que estes representam alocação de riscos ou a absorção de riscos no compartilhamento de recursos, permitindo ainda a coexistência de suas propriedades legais e informais, de forma a assegurar o comprometimento das partes para gerar benefícios mútuos (CASSON, 1997, p.7-9; GIBBONS, 2000; KREPS, 1990; WOLFF, 1996, p.102-105). Cada parte motivada a manter sua credibilidade e reputação com os outros agirá de forma cooperativa. Como Keefer e Knack (2005, p.706.) observam: "Quando as partes inerentemente confiam uma nas outras, as transações que requerem promessas confiáveis são mais fáceis de serem realizadas.” A condição básica para isso é a manutenção das expectativas de continuidade nas relações de vantagem mútua a longo prazo. Assim, a confiança torna-se essencial em contratos relacionais, pois, como argumenta Arrow (1969, p.62.), "com a ausência de confiança, seria muito custoso arranjar sanções e garantias alternativas, e muitas oportunidades benéficas de cooperação mútua seriam descartadas." A essência na abordagem da confiança em contratos relacionais está baseada no entendimento de que cada parte deve beneficiar-se do relacionamento. Em suma, a ausência de confiança pode significar a ausência de transações e de situações de benefício mútuo. Contratos relacionais envolvem investimentos no compartilhamento de comunicação aberta e informação sensível para a promoção de conhecimento complementar, em vez de cada parte investir em si mesmo. Nesse sentido, Macneil (1978, p.895.) ressalta duas normas relativas a contratos relacionais: a harmonização de conflitos internos e a preservação de relacionamentos.

A ausência de confiança em contratos de trabalho pode significar a ausência de níveis ótimos de esforços para se alcançar um nível de cooperação mais eficiente e, dessa forma, um ineficiente uso dos recursos. Quando soluções judiciais são substituídas por uma ordenação privada das relações contratuais, a confiabilidade das promessas necessita de comprometimentos confiáveis (credibilidade) que funcionam como o "cimento" dos contratos relacionais entre os indivíduos (FURUBOTN; RICHTER, 2001, p.276). Comprometimentos confiáveis envolvem atos de reciprocidade que resguardam uma relação bilateral quando estes assumem a forma de investimentos específicos e irreversíveis (WILLIAMSON, 1985, p.190-191.). Assim, podemos compreender que relações de confiança mútua podem ser usadas como um dispositivo de segurança para se construir comprometimentos confiáveis, como reputações, através de específicos investimentos ao longo do tempo, quando indivíduos começam a usar estratégias de reciprocidade (FURUBOTN; RICHTER, 2001, p.276-277; KREPS, 1990; WOLFF, 1996, p.103). Em Kreps (1990, p.125.) os efeitos de reputações são construídos sobre normas sociais, princípios de justiça e benefícios mútuos que, por sua vez, devem fornecer os indivíduos hierarquicamente inferiores de uma idéia sobre como a organização irá reagir em determinadas circunstâncias quando estas surgirem. Por fim, como afirma Williamson (1985, p. 247 e 249), esses princípios devem nortear comportamentos e expectativas dentro da empresa, promovendo um condicionamento social relativo à segurança no emprego e à proteção contra a exploração.

A confiança está relacionada a interações repetidas e a investimentos irreversíveis. Se essas experiências justificarem a confiança investida, elas se tornam mecanismos que determinam o cumprimento do contrato, atenuando as chances de oportunismo entre as partes. Um sistema de reputações é construído sobre relações de confiança ao longo do tempo, ao mesmo tempo em que é uma condição para o desenvolvimento de padrões de comportamento. Hardin (2002, p.145-150.) denomina feedback loop esse efeito da manutenção da confiança. 
Dessa forma, a confiança pode ser vista como uma forma racional de cooperação sob risco comportamental. E nesse sentido, empresas podem ser entendidas como redes de contratos relacionais entre os seus membros corporativos. De acordo com Furubotn e Richter (2001, p.266.), empresas são constituições sociais que envolvem, além de suas regras, investimentos no cultivo de relacionamentos entre indivíduos. Nesse sentido, os autores afirmam que o fortalecimento da cultura organizacional (confiança mútua) representa um importante objetivo para os investimentos realizados. Assim, os níveis de confiança dentro da empresa podem ser entendidos como indicadores da eficiência da gestão dos contratos relacionais. Uma das funções das redes de contratos relacionais baseadas na confiança mútua é a capacidade de subsistir as futuras incertezas do mercado. Como Furubotn e Richter (2001, p.277.) observam: "Elas podem ser usadas para superar as dificuldades impostas pela ausência, ou as limitações do mercado por futuros produtos. Elas se estendem em direção ao futuro e ajudam a reduzir a incerteza relacionada a mercados futuros. Elas facilitam a adaptação aos eventos imprevisíveis num mundo dirigido por mudanças, como aquelas que surgem com novos produtos, novos mercados, novas tecnologias de produção, novas formas organizacionais, e os resultados de eventos políticos e naturais. Elas contribuem para a formação racional de expectativas......e elas ajudam os tomadores de decisão a superarem problemas que surgem pela assimetria de informações."

Finalmente, vale observar que a opção pela adoção de um estilo de governança corporativa baseado em confiança possui alguns custos inerentes ao estabelecimento de um ambiente onde sejam criados e mantidos alguns elementos antecedentes e fundamentais para a construção de relacionamentos baseados em confiança. Em última análise, como afirma Hadfield (2005, p. 200.), o que define primordialmente essa relação de custo e eficiência são os diversos arranjos institucionais que se traduzem em incertezas ambientais.

\section{Relações entre confiança e desempenho organizacional}

Estudos em gestão têm buscado cada vez mais identificar formas eficazes de mensurar o desempenho organizacional. Por exemplo, Cannon, Achrol e Gundlach (2000) observam que as transações envolvendo interações dentro das organizações, também envolvem elementos intangíveis críticos que são raramente especificados. Baker, Gibbons e Murphy (1993) também observam que medidas objetivas de desempenho são raramente perfeitas e que a eficiência dos incentivos em contratos nas organizações depende muito de um conjunto de fatores sociais, psicológicos e econômicos. Assim, buscando observar os fatores de sucesso de arranjos organizacionais, faz-se necessário capturar esses fatores intangíveis ocultos que influenciam os relacionamentos em transações econômicas.

Uma série de fatores da cultura organizacional de uma empresa são relacionais e não estão escritos em nenhum lugar. Um mínimo de confiança é necessário para que o sistema possa funcionar; de outra forma é praticamente impossível que indivíduos possam tomar parte em qualquer transação que apresente intervalos de tempo na entrega de algum bem como fruto do trabalho especializado, ou para que indivíduos possam se beneficiar das trocas cooperativas necessárias ao trabalho especializado. Quanto maior forem os níveis de confiança dentro de um sistema, mais eficiente poderá ser o uso dos recursos existentes e maior será a probabilidade de ocorrerem ações de cooperação espontânea, reduzindo os custos de transação.

Para uma análise econômica mais adequada é importante considerar os níveis de confiança dentro das empresas como variáveis independentes que podem contribuir potencialmente para o desempenho organizacional. No entanto, a análise dos níveis de confiança isoladamente não é um determinante para o desempenho organizacional. Estudos empíricos frequientemente têm sugerido que a confiança possui um efeito mais moderador com o desempenho organizacional do que uma relação direta e positiva (ver DIRKS; FERRIN, 2001). Isso significa que níveis de confiança não determinam necessariamente um desempenho superior. Ou seja, mesmo que empresas apresentem baixos índices de confiança, isso não significa que irão apresentar necessariamente um baixo desempenho organizacional. É importante que uma analise dos níveis de confiança leve em consideração o contexto ou o ambiente institucional onde essas transações ocorrem, para que mecanismos alternativos de governança possam ser considerados. A noção de confiança traz em si a noção de risco comportamental associado, o que na perspectiva econômica significa custo associado (WOLFF, 2000, p.1.). O desenvolvimento da confiança consome tempo e requer investimentos específicos para a sua 
emergência e manutenção dentro do sistema. Para que uma empresa adote um estilo de gestão baseado em confiança faz-se necessário a manutenção de determinados elementos que alicerçam relacionamentos de confiança, tais como: normas de comportamento, conduta e punição, transparência organizacional e clareza na comunicação corporativa. Além disso, um ambiente de trabalho baseado em relações de confiança necessita de consecutivos investimentos na manutenção de uma relativa estabilidade das condições e perspectivas de trabalho. A percepção coletiva de justiça nos procedimentos, na mensuração e na distribuição dos resultados torna-se igualmente fator crítico para a gestão dos níveis de confiança. Whitener (1998) apresenta um estudo sobre alguns elementos diretamente relacionados à construção de ambientes que favoreçam relações de confiança. Esses elementos são: a qualidade da comunicação interna, a percepção de integridade, consistência e a preocupação com os empregados, além da delegação e do compartilhamento da autoridade. Esse conjunto de variáveis nos oferece, igualmente, uma melhor avaliação desses ambientes e da gestão dos bens intangíveis.

Tal estilo de gestão baseado em confiança pode ser altamente desejado para o cumprimento de determinadas tarefas organizacionais, mas o contexto institucional em que a empresa se encontra deve ser observado (Zanini, 2005 , p.17.). Se estilos de gestão baseados em confiança trazem vantagens potenciais, por outro lado, alguns investimentos são necessários. Ambientes de trabalho com uma atmosfera de "alta confiança" permitem que indivíduos possam realizar transações sem a necessidade de se precaverem contra eventuais comportamentos oportunistas dos outros, aceitando assim maiores riscos nessas transações. Em atmosferas de "baixa confiança" as pessoas serão mais receosas de realizarem investimentos de confiança uma nas outras porque elas percebem baixos níveis de motivação nos parceiros de interatividade em adotarem estratégias de reciprocidade, além de maior probabilidade de assumirem prejuízos pessoais nas transações. No entanto, devemos observar que, em específicos contextos organizacionais, pode ser possível que o fomento à competição interna entre os indivíduos membros da empresa seja mais eficiente e produza melhores resultados. Quando a eficiência das unidades de negócios está relacionada a um tipo de estratégia que incentiva a competição interna, esse processo permitirá a seleção de indivíduos e poderá privilegiar os esforços individuais em detrimento dos esforços coletivos (LAZEAR, 1998, p.226.). Em alguns casos, pode ser mais eficiente que baixos níveis de interatividade possam representar melhores resultados. Assim, o sistema de remuneração deverá privilegiar e premiar os esforços individuais dos trabalhadores. Como resultado, os trabalhadores poderão se dedicar mais e realizar maiores esforços para serem promovidos e alcançarem uma melhor remuneração. Nesses casos, investimentos na criação e na manutenção de um ambiente que estimule a confiança interpessoal entre os indivíduos pode não representar a forma mais eficiente de se obter resultados.

Por outro lado, nas organizações onde se estimula a competição interna entre os empregados, a tentativa da criação de laços pessoais de confiança pode ser extremamente difícil e lenta. Outras situações sugerem que o uso de controle explícito pode ser mais vantajoso do que contar com relações de confiança. Quando resultados são relativamente fáceis de serem mensurados, quando as tarefas possuem baixa complexidade e não demandam grande criatividade, velocidade de aprendizado, entendimento conceitual ou não exigem a transferência crítica do conhecimento (OUCHI, 1980, p.250-251; OSTERLOH; FREY, 2000, p.10.), a aplicação de mecanismos explícitos parece ser mais eficiente. No entanto, esse não parece ser o caso quando sistemas produtivos demandam o compartilhamento de informação sensível de forma a contribuir para a execução de tarefas especializadas e de alta complexidade e ganhos através da interatividade de grupos de trabalho (ADLER, 2001; OUCHI, 1980). Como afirmam alguns autores (ADLER, 2001, p.215; OSTERLOH; FREY, 2000, p.10), quando tarefas organizacionais são baseadas em inovação, criação e desenvolvimento de produtos, os melhores resultados poderão ser alcançados através da intensa interação entre as pessoas. Nesse sentido, Lazear (1998, p.261-262.) observa que a alta competitividade entre os empregados poderá ser um problema que afeta a atmosfera organizacional, o comportamento dos empregados e, conseqüentemente, a produtividade. Casos de alta competitividade entre indivíduos podem inibir significantemente a criação de relações de confiança e incentivar comportamentos oportunistas.

Nas situações em que o uso da confiança se torna um mecanismo de controle mais eficiente, observamos que, da mesma forma, o sistema de remuneração deverá estar alinhado com as estratégias de cooperação entre os empregados, incentivando investimentos pessoais de confiança. Nas circunstâncias onde a cooperação espontânea é um importante diferencial, as empresas podem optar por formas alternativas para afetar o 
comportamento dos empregados por meio de mudanças no sistema de remuneração, inibindo a competição entre pares nos grupos de trabalho.

Outro ponto importante para o privilégio de um estilo de gestão baseado em confiança é quando as medidas de desempenho tornam-se por demais ambíguas. Como Ouchi (1980, p.251.) comenta, nesses casos, o uso da confiança torna-se fundamental para o alinhamento dos objetivos individuais e organizacionais. Onde mecanismos burocráticos tradicionais baseados em controle explícito podem falhar, porque se torna impossível avaliar externamente o valor adicionado por indivíduo, o uso de confiança é mais recomendável. Nesse sentido, Ouchi (1980, p.250-251) e Dasgupta (2000, p.51.) observam que a possibilidade de monitorar as ações dos indivíduos é um fator crucial para se decidir entre mecanismos explícitos e confiança. De acordo com Gambetta (2000, p. 221.), a superioridade da confiança sobre mecanismos explícitos de controle e monitoração é provada, na medida em que é investida ainda antes da reação de quem se confia (B).

Alguns estudiosos observam que o intenso uso de mecanismos explícitos de controle, como monitoramento formal, apresentam grande probabilidade de aumentar as chances de oportunismo (AUKAKH; KOTABE; SAHAY, 1996; VAN DE VEN; WALKER, 1984); ou seja, a ênfase demasiada em garantias explícitas prejudica consideravelmente o desenvolvimento das relações de confiança. Prejudicar relações de confiança pode se traduzir em um alto custo porque, de modo inerente, o uso de confiança não requer despesas adicionais com garantias legais. Ademais, onde níveis de confiança se fazem presentes a necessidade de monitoração explícita e controle e os seus custos associados serão reduzidos. Além disso, o uso intensivo de mecanismos explícitos de controle envolve não somente investimentos em monitoração e controle formal, mas provavelmente uma perda considerável da motivação dos indivíduos para gerarem comprometimentos em direção ao atendimento dos objetivos organizacionais. Dessa forma, uma vez que contratos formais podem ser ineficientes (custosos, inerentemente incompletos, possivelmente inverificáveis e sujeitos às particularidades do sistema legal), eles podem ser usados somente em situações extremas para o atendimento das necessidades formais padronizadas do arcabouço legal.

Aqui vale entender que a presença de acordos formais, na forma de contratos explícitos, pode também funcionar para encorajar parceiros de interação a investirem confiança uns nos outros. Contratos de trabalho são exemplos que fornecem uma ilustração razoável dessas situações. Embora usualmente um contrato explícito deva ser assinado entre as partes, este não pode eliminar por completo o risco envolvido nas transações que irão ocorrer no futuro porque é impossível prever todas as contingências. No entanto, exatamente porque previamente um contrato formal é assinado entre as partes, um contrato relacional poderá se desenvolver. Em outras palavras, uma relação de confiança poderá ser iniciada, ou complementada, por um mecanismo formal. Nesse caso, o contrato formal funcionará apenas como uma garantia geral para estruturar e resguardar alguns princípios e expectativas gerais dentro do relacionamento, sem, no entanto prever todas as contingências futuras.

Empiricamente, encontraremos sempre a combinação e a coexistência de mecanismos explícitos e implícitos agindo em níveis diferentes dentro dos ambientes de negócios de forma complementar (BRADACH; ECCLES, 1998, p.277; OUCHI, 1980, p. 248). Por exemplo, Bradach e Eccles (1998, p.277 e 279) observam que a combinação de diferentes formas de coordenação - mercados, hierarquias e redes de relacionamentos baseadas em confiança - tem sido objeto de recentes estudos. Alguns elementos desses tipos ideais de estruturas de coordenação podem ser encontrados empiricamente, permitindo a formação de estruturas sociais colaterais. De forma particular, esses autores observam que grande parte das pesquisas aponta para a existência de relacionamentos estáveis construídos a longo prazo entre parceiros de interação independentes. Ouchi (1980, p.248.) nota que mecanismos explícitos e implícitos de controle podem ser encontrados com freqüência em condição complementar e em diferentes níveis, em qualquer organização real.

Analisando os estudos empíricos que abordam as relações de confiança entre indivíduos ou grupos e o desempenho organizacional, podemos observar a existência de uma relação positiva entre confiança e desempenho organizacional, ainda que não necessariamente direta. Estudos empíricos têm relacionado os efeitos positivos da confiança, por exemplo, no processo de transferência do conhecimento (ROBERTS, 2000; ROLLAND; CHAUVEL, 2000), para fortalecer o comprometimento dos empregados (BROCKNER et al, 
1997, PILLAI; SCHRIESHEIM; WILLIAMS 1991 UGBORO, 2003), e aumentar a eficiência e a produtividade organizacional (RING; VAN DE VEN, 1992, LANE; BACHMANN, 1998; SAKO, 1998).

Dirks e Ferrin (2001) apresentam uma série de estudos empíricos e apresentam evidências das relações de confiança e seus efeitos positivos nas atitudes e comportamentos dos indivíduos dentro das organizações, de forma a melhor compreender seus benefícios gerais. Esses autores focam sua atenção em pesquisas que apresentam medidas quantitativas de confiança, onde o elemento confiança é definido como um estado psicológico cognitivo (como definido neste artigo). A confiança é abordada como uma variável independente relacionada a outra variável dependente, indicando comportamento, desempenho, cognição ou atitude relativa ao trabalho. De acordo com esses autores, espera-se que níveis superiores de confiança resultem em atitudes mais positivas, altos níveis de comportamento cooperativo espontâneo e, conseqüentemente, num desempenho superior. Assim, Dirks e Ferrin (2001, p.453-454.) demonstram que a confiança está direta e positivamente relacionada à melhoria da comunicação interna, à aceitação de objetivos organizacionais e metas, a comportamentos relacionados ao conceito de cidadania corporativa, à satisfação e motivação dos empregados, à melhoria nos processos de negociação e redução de conflitos e finalmente, direta e indiretamente, ao aumento do desempenho individual e do desempenho de equipes de trabalho. A confiança é também observada por Aryee, Budhwar e Chen (2002) como um elemento mediador para a percepção coletiva de justiça distributiva e procedimental, satisfação do trabalho e intenções de turnover.

Outros estudos empíricos sobre equipes de trabalho virtuais revelam que devido à falta de comunicação física, à falta de suas propriedades verbais e não-verbais e de comunicação nas interações face a face elas apresentam níveis inferiores de confiança. Não surpreende que tais equipes de baixo nível de confiança apresentem igualmente menor produtividade (COUTU, 1998; JARVENPAA; KNOLL; LEIDNER, 1998). Embora sejam mais raros, outros estudos, como o de Langfred (2004), demonstram que altos índices de confiança sob determinadas circunstâncias de alta autonomia individual podem ser igualmente prejudiciais para o desempenho de equipes de trabalho (ver ZANINI, 2005, p.9.). Isso nos leva novamente à necessidade de analisar de forma não isolada os níveis de confiança dentro das organizações e a levar em consideração o contexto específico de análise, em conjunto com outros elementos da gestão organizacional.

\section{Conclusões}

Neste artigo buscamos adotar uma lógica funcional para as relações de confiança dentro das organizações. Abordamos algumas importantes propriedades dos relacionamentos de confiança. A confiança é relacional, no sentido de que a sua presença está baseada em normas e regras explícitas e implícitas de interação que são estabelecidas pelo ambiente organizacional. A confiança é cognitiva, no sentido de que os indivíduos podem calcular o risco comportamental relativo quando se envolvem em determinados relacionamentos.

Observamos que a confiança é um elemento fundamental para a coordenação implícita dentro das empresas e para a construção de bens intangíveis, principalmente, em determinados contextos organizacionais. Analisamos algumas propriedades do elemento confiança na primeira seção de forma a melhor compreender o seu funcionamento. Em seguida, analisamos o elemento confiança como central para os contratos relacionais - vide os contratos de trabalho - que combinam um mecanismo formal padronizado com uma relação contratual relacional que se desenvolve ao longo do tempo. Identificamos nesses contratos implícitos o elemento confiança como central para a sua coordenação e eficiência. Na ultima seção deste artigo analisamos a relação entre confiança e desempenho organizacional. Observamos que confiança não é uma variável determinante para o desempenho organizacional, mas é um elemento mediador que contribui implicitamente para uma maior eficiência na coordenação das diversas tarefas organizacionais.

Este artigo busca contribuir para os recentes estudos sobre as relações de confiança dentro das organizações, apresentando este elemento como central a ser analisado nos relacionamentos de trabalho. No entanto, a confiança é um elemento a ser ainda melhor compreendido e a sua relação com o desempenho organizacional inaugura um vasto campo para futuras pesquisas acadêmicas e para o desenvolvimento de uma inteligência corporativa mais eficiente na construção do capital intangível. 


\section{Referências}

ADLER, P. S. Market, hierarchy and trust: the knowledge economy and the future of capitalism. Organizational Science, v.12, n.2. p.215234, Mar./Apr. 2001.

ARROW, K. J. The organization of economic activity: issues pertinent to the choice of market versus non-market allocation. In: THE ANALYSIS and evaluation of public expenditure: the PPB system. U.S. Joint Economic Committee, 91st CONGRESS, 1st SESSION. Washington, DC: U.S. Government Printing Office, 1969. v.1. p.59-73.

. Information and economic behavior. Stockholm, Sweden: Federation of Swedish Industries, 1973.

ARYEE, S.; BUDHWAR, P.; CHEN, Z. X. Trust as a mediator of the relationship between organizational justice and work outcomes: test of a social exchange model. Journal of Organizational Behavior, n.23, p.267-285, 2002.

AUKAKH, P. S.; KOTABE, M.; SAHAY, A. Trust and performance in cross-border marketing partnerships: a behavioural approach. Journal of International Business Studies, special issue, p.1005-1032, 1996.

BAKER, G.; GIBBONS, R.; MURPHY, K. J. Relational contracts and the theory of the firm. Cambridge, MA: MIT, 1999. Unpublished manuscript.

BRADACH, J. L.; ECCLES, R. G. Price, Authority and Trust: from ideal types to plural forms, in: Markets, Hierarchies \& Networks - The Coordination of Social Life, Thompson, G. at all. (eds) Sage, London, p. 277-292, 1998.

BROCKNER, J. P. et al. When trust matters: the moderating affect of outcome favorability. Administrative Science Quarterly, n.42, p.558$583,1997$.

BULL, C. The existence of self-enforcing implicit contracts. The Quarterly Journal of Economics, p.147-160, Feb. 1987.

CANNON, J.; ACHROL, R.; GUNDLACH, G. Contracts, norms and plural form governance. Journal of the Academy of Marketing Science, p.180-194, Spring 2000.

CASSON, M. The economics of business culture - game theory, transaction costs and economic performance. Oxford: Clarendon Press, 1997.

COUTU, D. Trust in virtual teams. Harvard Business Review, Boston, p.20-21, May/June, 1998.

DASGUPTA, P. Trust as a commodity. In: GAMBETTA, D. (Ed.) Trust: making and breaking cooperative relations. Oxford: Department of Sociology, University of Oxford, 2000. chapter 4. p.49-72. Electronic edition.

DIRKS, K. T.; FERRIN, D. L. The role of trust in organizational settings. Organization Science, v.12, n.4, p.450-467, 2001.

FURUBOTN, E. G.; RICHTER, R. The new institutional economics: an assessment. In: (Ed.). The new institutional economics - a collection of articles from the Journal of Institutional and Theoretical Economics. Tübingen: J.C.B. Mohr, 1991.

of Michigan Press, 2001.

Institutions and economic theory - the contributions of the new institutional economics. Ann Arbor, MI.: The University

GAMBETTA, D. Can we trust trust? In: (Ed.). Trust: making and breaking cooperative relations. Oxford: Department of Sociology, University of Oxford, 2000. chapter 13. p.213-237. Electronic edition.

GIBBONS, R. Trust in social structures: Hobbes and Coase meet repeated games. In: C0OK, K. (Ed.). Trust in society. New York: Russell Sage Foundation, 2000.

Relational contracts and the theory of the firm. [S.I.]: MIT Sloan School and NBER, 2001. Working paper.

HADFIELD, G. K. The many legal institutions that support contractual commitments. In: MÉNARD, C.; SHIRLEY, M. M. (Ed.). Handbook of New Institutional Economics. New York: Springer, 2005. p.175-204.

HARDIN, R. Trust in government. In: BRAITHWAITE, V.; LEVI, M. (Ed.). Trust and governance. New York: Russell Sage Foundation, 1998. v.1. p.9-27. (Series of Trust).

Trust and trustworthiness. New York: Russell Sage Foundation, 2002.

JARVENPAA, S. L.; KNOLL, K.; LEIDNER, D. E. Is anybody out there? Antecedents of trust in global virtual teams. Journal of Management Information Systems, v.14, n.4, p.29-64, 1998.

KEEFER, P.; KNACK, S. Social capital, social norms and the new institutional economics. In: MÉNARD, C.; SHIRLEY, M. M. (Ed.). Handbook of New Institutional Economics. New York: Springer, 2005. p.701-726. 
KREPS, D. M. Corporate culture and economic theory. In: PERSPECTIVES on positive political economy. Cambridge: Cambridge University Press, 1990.

LANE, C; BACHMANN, R. Trust within and between organizations - conceptual issues and empirical applications [S.I.]: Oxford University Press, 1998.

LANGFRED, C. W. Too much of a good thing? Negative effects of high trust and individual autonomy in self-managing teams. Academy of Management Journal, v.47, n.3, p.385-399, June 2004.

LAZEAR, E. P. Personnel economics for managers. New York, NY: Wiley, 1998.

MACAULEY, S. Non-contractual relations in business: a primarily study. American Sociology Review, n.28, p.55-67, 1963.

MACNEIL, I. Contracts: adjustment of long-term economic relations under classical, neo-classical and relational contract law. Northwestern Law Review, n.72, p.854-906, 1978.

OSTERLOH, M.; FREY, B. S. Motivation, knowledge transfer and organizational forms. Organization Science, v.11, n.5, p.538-550, Sept./Oct. 2000.

OUCHI, W. G. Markets bureaucracies and clans. Administrative Science Quarterly, n.25, p.129-141, 1980.

PILLAI, R. C.; SCHRIESHEIM, C.; WILLIAMS, E. Fairness perceptions and trust as mediators for transformational and transactional leadership: a two-study sample. Journal of Management, n.25, p.897-933, 1991.

RING, P. S.; VAN DE VEN, A. H. Structuring cooperative relationships between organizations. Strategic Management Journal, n.13, p.483498, 1992

RIPPERGER, T. Ökonomik des Vertauens. Tübingen: [s.n.], 1998.

ROBERTS, J. From know-how to show-how? Questioning the role of information and communication technologies in knowledge transfer. Technology Analysis and Strategic Management, 12, 4, pp. 429-443, 2000.

ROBERTS, J.; VAN DEN STEEN, E. Human capital and corporate governance. In: SCHWALBACH, J. (Ed.). Corporate governance essays in honor of Horst Albach. [S.I.]: Springer, 2001. p.128-143.

ROLLAND, N.; CHAUVEL, D. Knowledge transfer in strategic alliances. In: DESPRES, C.; CHAUVEL, D. (Ed.). Knowledge horizons: the present and the promise of knowledge management. Boston, MA: Butterworth Heinemann, 2000. p.225-236.

SAKO, M. Does trust improve business performance? In: LANE, C; BACHMANN, R. (Ed.). Trust within and between organizations. Oxford: Oxford University Press, 1998.

SIMON, H.A. A formal theory of the employment relationship. Econometrica, Journal of the Econometric Society, v.19, n.3, July 1951.

UGBORO, I. 0. Influence of managerial trust on survivors' perceptions of job insecurity and organizational commitment in a post restructuring and downsizing environment. Journal of Behavioral and Applied Management, v.4, n.3, p.231-253, Winter 2003.

VAN DE VEN, A. H.; WALKER, G. The dynamics of interorganizational coordination. Administrative Science Quarterly, n.29, p.598-621, 1984.

WHITENER, E. M. et al. Managers as initiators of trust: an exchange relationship framework for understanding managerial trustworthy behavior. Academy of Management Review, v.23, p.513-530, 1998.

WILLIAMSON, O. E. The economic institutions of capitalism - firms, markets, relational contracting. New York: Free Press, 1985.

WOLFF, B. Contractual problems in market relations. In: BERNITZ, U.; HALLSTÖM, P. (Hg.) Principles of Justice and the European Union. Stockholm: Juristförlaget, 1995. p.83-95.

Constitutional contracting and corporate constitution. In: PICOT, A.; SCHLICHT, E. (Hg.): Perspectives on contract theory.[S.I.]: Springer, 1996. p.95-108.

Kalkül und Vertrauen - Zur Institutionalisierung von Moral in der Ökonomik. Vertrauen in Gesellschaft und Organisationen, Tutzing, 20, Mai 2000. (Manuskript zum Vortrag im Rahmen der Tagung).

ZANINI, M. T. F. Relações de confiança nas empresas da nova economia informacional - uma avaliação dos efeitos da incerteza sobre 0 comportamento organizacional. Cadernos Ebape.Br, n.4, Dez. 2005. 
${ }^{1}$ Gibbons (2001) observa que contratos relacionais são denominados por vezes "self-enforcing", "implícitos" ou ambos. O uso do termo "relacional" segue a terminologia da literatura legal, particularmente, Macneil (1978). Para definições originais sobre contrato relacional na perspectiva econômica, ver Simon (1951) e Bull (1987).

${ }^{2}$ Bull (1987, p.149.) usa a seguinte definição baseada na teoria dos jogos para contratos implícitos: "um contrato implícito é mais um acordo não-contratual que corresponde a um equilíbrio de Nash para os jogos interativos bilaterais repetidos, do que um acordo degenerado em uma seqüência de equilíbrios de Nash para jogos transacionais, de um só lance." 